Mayne Thaiane Azevedo de Jesus (iD) https://orcid.org/0000-0003-0551-9509

Silvia Ferrite

(iD) http://orcid.org/0000-0001-5344-3751

Tânia Maria de Araújo ${ }^{b}$

(D) https://orcid.org/0000-0003-2766-7799

Maria Lúcia Vaz Masson ${ }^{\mathrm{a}}$

(iD) https://orcid.org/0000-0003-0733-1753

a Universidade Federal da Bahia (UFBA), Instituto de Ciências da Saúde, Departamento de Fonoaudiologia. Salvador, BA, Brasil.

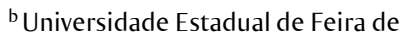
Santana (UEFS), Departamento de Saúde. Feira de Santana, BA, Brasil.

Contato:

Maria Lúcia Vaz Masson

E-mail:

masson@ufba.br

Os autores declaram que o trabalho foi subvencionado pela Bolsa Iniciação Científica PIBIC/FAPESB (nº BOL 2021/2016). Projeto: "Não há evidências suficientes para reconhecer o distúrbio de voz como doença relacionada ao trabalho?"

Os autores declaram que não há conflitos de interesses.

Os autores informam que o trabalho foi baseado em trabalho de conclusão de curso de graduação (TCC) de Mayne Thaiane Azevedo de Jesus, intitulado Distúrbio de Voz Relacionado ao Trabalho: Revisão Integrativa, em 2017, no Curso de Graduação em Fonoaudiologia da Universidade Federal da Bahia; e que foi apresentado no XXV Congresso Brasileiro de Fonoaudiologia, 2017, e no Congresso de Pesquisa, Ensino e Extensão da Universidade Federal da Bahia, 2017. Salvador, BA.

Recebido: 30/10/2018

Revisado: 12/04/2019

Aprovado: 29/05/2019

\section{Distúrbio de voz relacionado ao trabalho: revisão integrativa}

\author{
Work-related voice disorder: an integrative review
}

\section{Resumo}

Objetivos: identificar e sumarizar evidências de estudos sobre a associação entre trabalho e distúrbio de voz. Métodos: revisão integrativa da literatura publicada nas bases de dados PubMed e SciELO entre 2003 e 2017. Foram elegíveis estudos observacionais, em português ou inglês, disponíveis na íntegra para consulta, que abordassem a frequência de disfonia (prevalência ou incidência). Resultados: foram analisados 47 artigos, a idade media sumarizada dos participantes era de 40,6 anos, 78,7\% dos artigos estudaram ambos os sexos e $80,9 \%$ pesquisaram professores. A prevalência média de distúrbios da voz associados ao trabalho foi de $44,2 \%$ e a incidência de $17,0 \%$. O ruído foi indicado como fator associado à disfonia em $25,5 \%$ dos estudos, seguido da carga horária de trabalho extensa (17,0\%) e alergias (14,9\%). Conclusão: os resultados indicam forte conexão entre distúrbio de voz e trabalho, confirmando a elevada prevalência de disfonia, especialmente em trabalhadores que utilizam a voz profissionalmente, bem como sua associação com fatores da organização e do ambiente de trabalho, contribuindo para: a) fortalecer o reconhecimento formal do distúrbio de voz relacionado ao trabalho; b) oferecer embasamento técnico à legislação; c) reforçar a premência de uma política pública para proteção vocal dos trabalhadores.

Palavras-chave: distúrbios da voz; ambiente de trabalho; condições de trabalho; riscos ocupacionais; saúde do trabalhador.

\begin{abstract}
Objectives: to identify and summarize evidence from studies on the association between work and voice disorder. Methods: integrative review of the literature published in PubMed and SciELO between 2003 and 2017. We included open access observational studies in Portuguese or English concerning frequency of dysphonia (prevalence or incidence). Results: we analyzed 47 articles. The participants' average age was 40.6 years, 78.7 of the articles studied both sexes and $80.9 \%$ investigated teachers. The mean prevalence of work-related voice disorder was $44.2 \%$ and the incidence of $17.0 \%$. Noise was pointed out as an associated factor of dysphonia in $25.5 \%$ of the studies, followed by long work hours (17.0\%) and allergies (14.9\%). Conclusion: the results show a strong association between voice disorders and work, confirming a high prevalence of dysphonia especially in workers who use their voice professionally, as well as its association to job organization and work environment, contributing to: a) strengthen the formal acknowledgment of work-related voice disorder; b) provide technical grounds to law; c) to reinforce the urgency of public policies to protect the workers' voice.
\end{abstract}

Keywords: voice disorders; work environment; labor conditions; occupational risks; occupational health. 


\section{Introdução}

Segundo a Organização Mundial da Saúde (OMS), aproximadamente $45 \%$ da população mundial está inserida em alguma atividade profissional ${ }^{1}$. Pesquisas em Saúde do Trabalhador visam compreender as relações entre o trabalho e o processo saúde/doença. A Política Nacional de Saúde do Trabalhador e da Trabalhadora tem a finalidade de desenvolver a integralidade do cuidado à saúde, com ênfase na vigilância, promoção, proteção da saúde dos trabalhadores e redução da morbimortalidade decorrente dos processos produtivos ${ }^{2}$. A Lei $\mathrm{n}^{0}$ 8080/90 já previa, em seu artigo $2^{\circ}$, que a Saúde do Trabalhador é um conjunto de serviços voltados à promoção e proteção da saúde, por meio de ações de vigilância epidemiológica e sanitária, bem como à recuperação e reabilitação dos trabalhadores submetidos a riscos e agravos decorrentes das condições de trabalho ${ }^{3}$.

Dentre as doenças ocupacionais, o distúrbio de voz relacionado ao trabalho (DVRT) apresenta evolução insidiosa, lenta e progressiva. Está relacionado às condições de ambiente e organização do trabalho ${ }^{4}$, assim como categorias que utilizam a voz profissionalmente, a exemplo de professores, teleoperadores, cantores, atores e locutores ${ }^{5}$. Registra-se, nos últimos anos, aumento da incidência de agravos vocais, especialmente entre os profissionais que utilizam a voz como instrumento de trabalho ${ }^{4}$.

No caso específico da relação entre voz e trabalho, deve-se destacar as discussões e debates ocorridos a partir de 1997, em seminários promovidos pela Pontifícia Universidade Católica de São Paulo (PUC-SP), envolvendo o Centro de Referência em Saúde do Trabalhador (CEREST/SP), o Conselho Regional de Fonoaudiologia (CFFa.), a Sociedade Brasileira de Fonoaudiologia (SBFa.) e entidades representantes de trabalhadores, nominalmente os sindicatos de radialistas, professores, teleoperadores e atores ${ }^{6-8}$.

O DVRT pode ser definido como uma alteração vocal, associada à atividade profissional, que impacta a comunicação e atuação do trabalhador, haja ou não lesão na laringe ${ }^{9}$. O desenvolvimento do DVRT está associado ao processo de trabalho, no que tange aos fatores organizacionais e ambientais, assim como fatores pessoais predisponentes (idade, sexo ou problemas de saúde em geral), conforme definido no Protocolo DVRT ${ }^{4}$.

Para avaliar a relação entre trabalho e adoecimento, em 1984, Schilling sistematizou uma classificação segundo a qual a doença do trabalhador é provocada pela atividade profissional em diferentes níveis: I) causa única; II) fator contributivo para o estabelecimento da doença; ou III) fator agravante de condição pré-existente ${ }^{10}$. O DVRT está nos grupos II e III da Classificação Schilling, devido a sua natureza multicausal ${ }^{11}$.
No Brasil, a definição de doenças relacionadas ao trabalho (DRT) é uma competência do Ministério da Saúde, estabelecida pela Lei $8080 / 90^{3}$ e Portaria GM/MS n ${ }^{\circ}$ 1.339/99, de 18 de novembro de 1999. Esta definição foi apresentada em lista que associava fatores de risco de natureza ocupacional com as respectivas doenças, segundo a Classificação Estatística Internacional de Doenças e Problemas Relacionados com a Saúde (CID-10). O distúrbio de voz não está nessa "lista-mãe" ${ }^{8}$. É apresentado posteriormente em manual técnico específico ${ }^{12}$. Embora sejam independentes, as listas de agravos relacionados ao trabalho do Ministério da Saúde e da Previdência Social têm origem nessa mesma "lista-mãe", da qual derivam outras listas específicas para fins de notificação compulsória (a Portaria MS/GM nº 204/16, última publicada, não incluía o DVRT) e concessão de benefícios da Previdência Social, estabelecida pelo Decreto $\mathrm{n}^{0} 3.048 / 99^{8}$. Mesmo que não esteja na lista de notificação compulsória, há iniciativas, a exemplo dos estados do Rio de Janeiro e Alagoas e município de Niterói/RJ, os quais, com base no artigo 10 da Portaria MS/GM no $104 / 11$, que facultava a elaboração de listas específicas, segundo o perfil epidemiológico local, iniciaram a notificação do DVRT ${ }^{8}$.

A ausência do reconhecimento formal do DVRT gera dificuldades na notificação pelo Sistema de Informação de Agravos de Notificação (SINAN) em termos de abrangência, comprometendo a identificação do adoecimento em trabalhadores, a estimativa de prevalência e o planejamento de políticas públicas para o seu manejo ${ }^{8}$. Além disso, a inexistência deste reconhecimento dificulta a tomada de decisões periciais a favor do trabalhador, podendo comprometer o acesso ao benefício-acidente do Instituto Nacional do Seguro Social (INSS) em casos de problemas de voz, uma vez que não há normativa que reconheça formalmente o DVRT. Sua inclusão na lista de doenças relacionadas ao trabalho possibilitaria estabelecer o nexo causal mais facilmente, sem a necessidade de avaliação caso a caso ${ }^{8}$.

Em abril de 2017, a revisão do Protocolo DVRT ${ }^{4}$ foi retomada pelo Ministério da Saúde, que o publicou oficialmente em 31 de julho de 2018. Apesar dos esforços mais recentes, ainda há o desafio da implementação do protocolo e da inclusão do DVRT na lista de doenças relacionadas ao trabalho. A sistematização de evidências empíricas do nexo causal entre distúrbio de voz e trabalho ainda é necessária, de modo a fortalecer o conhecimento sobre esse crescente problema de Saúde Pública.

Estudos de revisão de literatura publicados na década de 2000 destacaram a fragilidade metodológica das pesquisas até então realizadas, dificultando a consolidação da relação entre trabalho e problemas de voz ${ }^{13,14}$. Portanto, é necessário avaliar essas investigações e compará-las, destacando suas concordâncias e contradições, potenciais e limitações, 
de modo a estabelecer um conjunto consistente de conhecimentos sobre a temática.

Diante do exposto, este artigo pretende identificar e sumarizar as evidências de pesquisas que focalizaram a relação entre trabalho e distúrbio de voz, de modo a contribuir para estabelecer o nexo entre eles.

\section{Métodos}

Realizou-se estudo de revisão integrativa de literatura, utilizando descritores e palavras-chave em inglês, combinados aos operadores booleanos "and" e "or", da seguinte maneira: (voice disorder or dysphonia or voice loss or vocal load or voice loading) and (occupational or work related or occupational health or risk) and (teacher or faculty or singer or priests or pastor or aerobics instructors or call center or telemarketer or seller or actor). Optou-se por realizar a busca em língua inglesa em razão da maior quantidade de referências em comparação com a língua portuguesa. As bases de dados selecionadas foram PubMed e SciELO, no período de 2003 a 2017. O marco inicial em 2003 justifica-se pela publicação da primeira revisão de literatura que alertava sobre a fragilidade das evidências da relação entre distúrbio de voz e trabalho ${ }^{13}$.

Os critérios de inclusão foram: artigos publicados em português ou inglês, no período estabelecido, de caráter observacional, disponíveis na íntegra para consulta e que apresentassem medidas de frequência (prevalência ou incidência) de disfonia/agravos vocais e/ou fatores associados. Excluíram-se os artigos não relacionados ao tema DVRT.

A partir do levantamento conduzido, elaborou-se banco de dados específico, em planilha do programa Microsoft Excel, com as seguintes variáveis de interesse: ano de publicação, periódico, desenho de estudo, número de sujeitos, dados sociodemográficos da população estudada (sexo, idade, profissão, país de estudo e etapa de ensino em estudos com professores), métodos de análise utilizados para identificar os distúrbios de voz e dados epidemiológicos sobre o DVRT (prevalência, incidência e fatores associados à alteração vocal). A variável ano de publicação foi categorizada em faixas de 4 a 5 anos (2005 a 2009, 2010 a 2013, 2014 a 2017). O tipo de desenho de estudo foi categorizado em: transversal, caso-controle, híbrido (seguimento e seccional e transversal/intervenção) ${ }^{15}$. As variáveis que registraram a frequência de até dois eventos foram informadas como "outros/outras".

Os dados foram analisados de maneira descritiva, utilizando o programa Statistical Package for Social Sciences (SPSS), versão 20, sendo expressos em número absoluto e frequência, para as variáveis categóricas; médias sumarizadas, desvio-padrão, valores médios, mínimo e máximo, para as variáveis contínuas. Optou-se por extrair a mediana da média do número de sujeitos devido à grande variação nos diferentes estudos. Para a variável idade, foi obtida a média sumarizada, desconsiderando-se aquelas expressas em termos de faixa.

\section{Resultados}

Foram encontrados, no total, 317 artigos utilizando a combinação de palavras-chave e descritores nas bases PubMed e SciELO. Desse total, 47 artigos foram eleitos para este estudo, de acordo com os critérios de inclusão estabelecidos (Figura 1).
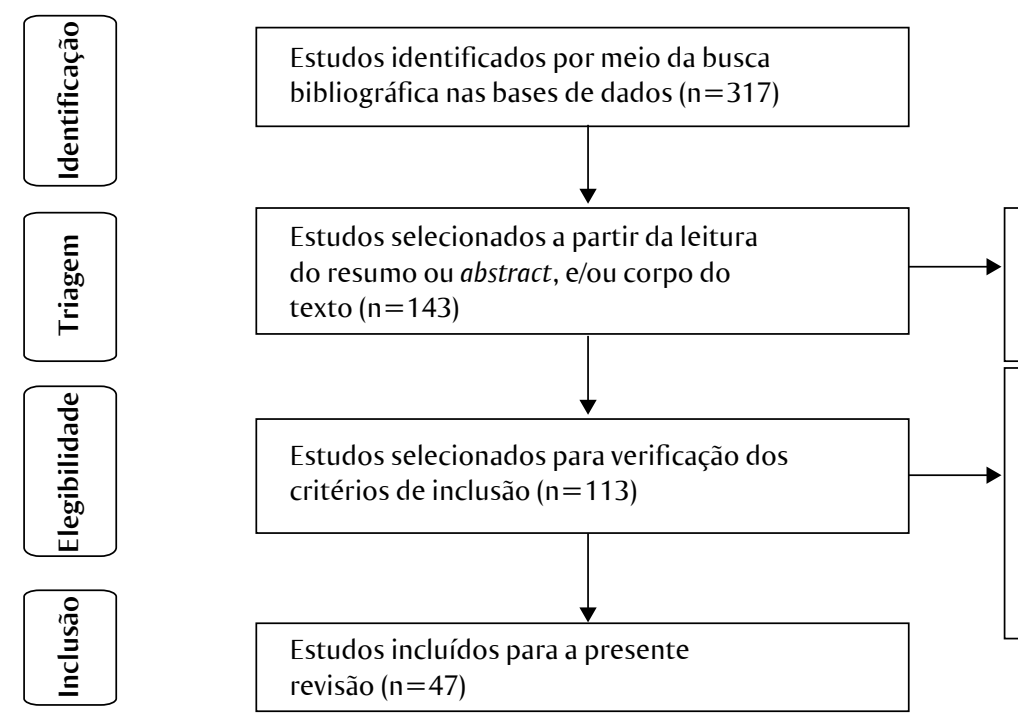

Estudos excluídos por duplicidade e/ou por não atenderem estritamente a temática estudada $(n=30)$

Estudos excluídos $(n=66)$

- 15 revisões de literatura

- 21 artigos não estavam disponíveis na íntegra

- 28 estudos não observacionais

- 2 não forneciam dados sobre prevalência, incidência e fatores associados

Figura 1 Fluxograma do resultado da busca nas fontes de informações, seleção e inclusão dos artigos na revisão 
A maior parte das pesquisas foi realizada considerando homens e mulheres conjuntamente $(78,7 \%)$, entre os anos de 2010 e 2013, sendo o primeiro artigo encontrado em 2005. O periódico com mais estudos publicados foi o Journal of Voice $(46,8 \%)$. Em relação aos desenhos de estudo, os transversais predominaram $(87,2 \%)$, sendo os docentes a categoria mais estudada $(80,9 \%)$. Outras profissões como cantor, instrutor de fitness, ator e metalúrgico foram investigadas em apenas um estudo cada $(2,1 \%)$. Dentre os fatores ambientais ressalta-se o ruído $(25,5 \%)$ e, dentre os organizacionais, a extensa carga horária $(17,0 \%)$. As alergias $(14,9 \%)$ foram o fator pessoal relatado mais frequentemente.

Em relação à etapa de ensino, professores do ensino fundamental I e II foram alvo da maioria das pesquisas nesta categoria $(26,3 \%)$, provavelmente devido a sua atuação em escolas municipais, que compreendem apenas o ensino fundamental.

Nesta revisão, todos os estudos estimaram a prevalência/incidência de distúrbio de voz com base na autorreferência, por meio de questionários ou protocolos de autoavaliação. Utilizou também avaliação perceptivo-auditiva $(14,8 \%)$ e otorrinolaringológica $(8,5 \%)$.

O Brasil foi o país com maior número de estudos, com 59,6\% das pesquisas identificadas, seguido por Colômbia, Estados Unidos e Finlândia, ambos com $4,3 \%$ cada.

A Tabela 1 corresponde às variáveis qualitativas referentes ao ano de publicação, periódico, desenho de estudo, população, tipos de avaliação, local do estudo e etapa de ensino, no caso de professores.

Tabela 1 Variáveis qualitativas relacionadas às características das publicações e aspectos metodológicos dos artigos encontrados

\begin{tabular}{|c|c|c|}
\hline Variável & $n$ & $\%$ \\
\hline \multicolumn{3}{|l|}{ Ano de publicação } \\
\hline $2005-2009$ & 8 & 17,0 \\
\hline $2010-2013$ & 22 & 46,8 \\
\hline 2014-2017 & 17 & 36,2 \\
\hline \multicolumn{3}{|l|}{ Periódicos } \\
\hline Audiology-Communication Research & 3 & 6,5 \\
\hline Cad. Saúde Pública & 3 & 6,4 \\
\hline CEFAC & 2 & 4,3 \\
\hline CoDAS & 3 & 6,4 \\
\hline Journal of Voice & 22 & 46,8 \\
\hline Occup Med (Lond) & 2 & 4,3 \\
\hline Rev. Saúde Pública & 3 & 6,4 \\
\hline Outras* & 9 & 18,9 \\
\hline \multicolumn{3}{|l|}{ Desenho do estudo } \\
\hline Transversal & 41 & 87,2 \\
\hline Caso-controle & 3 & 6,4 \\
\hline Coorte & 1 & 2,1 \\
\hline Híbrido & 2 & 4,3 \\
\hline \multicolumn{3}{|l|}{ População } \\
\hline Professor & 38 & 80,9 \\
\hline Padre & 3 & 6,4 \\
\hline Call Center & 2 & 4,3 \\
\hline Outros* & 4 & 8,4 \\
\hline \multicolumn{3}{|l|}{ Sexo } \\
\hline Feminino & 7 & 14,9 \\
\hline Masculino & 3 & 6,4 \\
\hline Ambos & 37 & 78,7 \\
\hline
\end{tabular}

(Continua) 
Tabela 1 Continuação...

\begin{tabular}{|c|c|c|}
\hline Variável & $n$ & $\%$ \\
\hline \multicolumn{3}{|l|}{ Instrumento } \\
\hline Questionário & 30 & 63,8 \\
\hline Protocolo & 17 & 36,2 \\
\hline \multicolumn{3}{|l|}{ Tipo de avaliação } \\
\hline Autoavaliação**** & 47 & 100,0 \\
\hline Perceptivo-auditiva & 7 & 14,8 \\
\hline Análise acústica & 5 & 10,7 \\
\hline Laringoscopia & 4 & 8,5 \\
\hline Mensuração de ruído & 2 & 4,3 \\
\hline \multicolumn{3}{|l|}{ País de Estudo } \\
\hline Brasil & 28 & 59,6 \\
\hline Colômbia & 2 & 4,3 \\
\hline Estados Unidos & 2 & 4,3 \\
\hline Finlândia & 2 & 4,3 \\
\hline Outros* & 13 & 27,7 \\
\hline \multicolumn{3}{|l|}{ Estado do Brasil $(\mathrm{n}=28)$} \\
\hline Bahia & 4 & 14,3 \\
\hline Minas Gerais & 4 & 14,3 \\
\hline Paraíba & 2 & 7,1 \\
\hline Rio Grande do Sul & 3 & 10,7 \\
\hline São Paulo & 12 & 42,9 \\
\hline Outros* & 3 & 10,7 \\
\hline \multicolumn{3}{|l|}{ Municípios do Brasil $(\mathrm{n}=28)$} \\
\hline Belo Horizonte & 3 & 10,7 \\
\hline João Pessoa & 2 & 7,1 \\
\hline Pelotas & 2 & 7,1 \\
\hline Salvador & 3 & 10,7 \\
\hline São Paulo & 8 & 28,6 \\
\hline Outros* & 10 & 35,8 \\
\hline \multicolumn{3}{|l|}{ Etapa de ensino dos estudos com professores $(n=38)$} \\
\hline Fundamental I e II & 10 & 26,3 \\
\hline Fundamental I & 8 & 21,1 \\
\hline Fundamental I e II e ensino médio & 5 & 13,1 \\
\hline Pré-escola, fundamental I e II e ensino médio & 2 & 5,3 \\
\hline $\begin{array}{l}\text { Pré-escola e fundamental I } \\
\text { Fundamental II }\end{array}$ & $\begin{array}{l}2 \\
2\end{array}$ & $\begin{array}{l}5,3 \\
5,3\end{array}$ \\
\hline Outros* & 4 & 10,6 \\
\hline Não informaram & 5 & 13,1 \\
\hline
\end{tabular}

*A categoria "outros/outras" contemplou publicações que tiveram apenas um evento citado (2,1\%). Periódicos: PLOS ONE, Acta Otorhinolaryngol. Ital., J. Appl. Oral Sci., Rev. Bras. Epidemiol., Occup. Med. (Lond)., Rev. Dist. Com., Arq. Int. Otorrinolaringol., Rev. Bras. Saúde Ocup., BMC Public Health e Wiener klinische Wochenschrift. População do estudo: trabalhador metalúrgico, ator, instrutor fitness e cantor. Países: Austrália, Bélgica, Egito, Eslovênia, Espanha, França, Índia, Itália, Malásia, Nigéria, Nova Zelândia, Singapura e Taiwan. Estados: Paraná, Santa Catarina e Alagoas. Municípios do Brasil: Bauru, Betim, Botucatu, Florianópolis, Londrina, Maceió, Piracicaba, Porto Alegre, Salto e Vitória da Conquista. Etapa de ensino: Fundamental II e médio; Pré-escola; Pré-escola, fundamental I, II e educação especial; Pré-escola, fundamental I e II.

***: Todos os artigos referiram autoavaliação. As outras modalidades são adicionais a esta, sendo referidas conforme citadas. Por isso o percentual, se somado, é superior a $100 \%$. 
Na Tabela 2 apresentam-se as variáveis quantitativas referentes ao número de sujeitos, idade, prevalência e incidência de alteração vocal das publicações encontradas. Foram considerados 28 artigos, os quais forneceram a média de idade (40,6 anos). Outros estudos $(n=17)$ apresentaram as faixas de idade enquanto duas pesquisas não referiram a idade dos participantes, sendo excluídas do cálculo. Predominaram estudos de corte transversal com informações sobre prevalência de alteração vocal média de $44,2 \%$, seguidos por caso-controle, com média de $65,0 \%$ para os casos, e $34,3 \%$ para os controles e um único estudo de coorte, com incidência de $17,0 \%$. Os estudos híbridos não foram considerados para esta análise.

Dentre os artigos estudados, $63,8 \%$ analisaram fatores organizacionais, do processo de trabalho e ambientais associados à alteração vocal, entre os quais os fatores mais citados foram extensa carga horária (17\%), número de alunos por turma e contato com poeira, giz e fumaça, ambos com $12,8 \%$, conforme apresentados na Tabela 3.

Na Tabela 4, apresentam-se os aspectos individuais associados à alteração vocal, analisados em $42,6 \%$ dos artigos, predominando as alergias $(14,9 \%)$ e infecção do trato respiratório $(12,8 \%)$.

Tabela 2 Sumarização das variáveis quantitativas referentes ao número de sujeitos, idade, prevalência e incidência de alteração vocal nas publicações encontradas

\begin{tabular}{lcc}
\hline \multicolumn{1}{c}{ Variável } & Média & Mínima-Máxima \\
\hline Número de sujeitos $(\mathrm{n}=47)$ & - & $13-6.039$ \\
Idade $(\mathrm{n}=28)$ & 40,6 & $25,0-52,0$ \\
Prevalência $(\mathrm{n}=41)$ & 44,2 & $10,0-87,0$ \\
Prevalência caso $(\mathrm{n}=3)$ & 65,0 & $27,5-93,4$ \\
Prevalência controle $(\mathrm{n}=3)$ & 34,3 & $7,5-51,0$ \\
Incidência $(\mathrm{n}=1)$ & 17,0 & - \\
\hline
\end{tabular}

Prevalência $(n=41)$ refere-se aos estudos de corte transversal. Prevalência caso $(n=3)$ e Prevalência controle $(n=3)$ referem-se a estudos caso-controle. Incidência $(n=1)$ refere-se a estudo de coorte. Não foram considerados os estudos híbridos $(n=2)$ para esta análise.

Tabela 3 Fatores de ambiente e organização do trabalho associados à alteração vocal nas publicações encontradas

\begin{tabular}{|c|c|c|}
\hline Variável & $n$ & $\%$ \\
\hline \multicolumn{3}{|l|}{ Organização do trabalho } \\
\hline Extensa carga horária & 8 & 17,0 \\
\hline Número de alunos na turma & 6 & 12,8 \\
\hline Uso excessivo da voz & 4 & 8,5 \\
\hline Tempo de trabalho & 4 & 8,5 \\
\hline Ameaça no trabalho & 3 & 6,4 \\
\hline Desgaste frequente com alunos & 3 & 6,4 \\
\hline Falar alto & 3 & 6,4 \\
\hline Altas demandas físicas & 2 & 4,3 \\
\hline Ausência de repouso vocal & 2 & 4,3 \\
\hline Fiscalização constante/pressão & 2 & 4,3 \\
\hline Insulto & 2 & 4,3 \\
\hline Ritmo de trabalho estressante & 2 & 4,3 \\
\hline Assédio & 1 & 2,1 \\
\hline Agressão & 1 & 2,1 \\
\hline Autonomia & 1 & 2,1 \\
\hline Benefício de saúde & 1 & 2,1 \\
\hline Criatividade & 1 & 2,1 \\
\hline Difícil acesso a água & 1 & 2,1 \\
\hline Disciplina & 1 & 2,1 \\
\hline Equilíbrio & 1 & 2,1 \\
\hline
\end{tabular}


Tabela 3 Continuação...

\begin{tabular}{|c|c|c|}
\hline Variável & $n$ & $\%$ \\
\hline Grau de ensino & 1 & 2,1 \\
\hline Mau comportamento de aluno & 1 & 2,1 \\
\hline Microfone (fator de proteção) & 1 & 2,1 \\
\hline Opinião & 1 & 2,1 \\
\hline Preconceito/racismo & 1 & 2,1 \\
\hline Realização profissional & 1 & 2,1 \\
\hline Remuneração & 1 & 2,1 \\
\hline Risco biológico & 1 & 2,1 \\
\hline Uso da voz fora do trabalho & 1 & 2,1 \\
\hline Violência & 1 & 2,1 \\
\hline \multicolumn{3}{|l|}{ Ambiente de trabalho } \\
\hline Ruído & 12 & 25,5 \\
\hline Poeira/giz/fumaça & 6 & 12,8 \\
\hline Acústica do ambiente & 5 & 10,6 \\
\hline Ar seco & 3 & 6,4 \\
\hline Limpeza precária & 3 & 6,4 \\
\hline Ar frio & 2 & 4,3 \\
\hline Produtos irritativos & 2 & 4,3 \\
\hline Recursos e equipamentos insuficientes & 2 & 4,3 \\
\hline Distância do público & 1 & 2,1 \\
\hline Mudança de temperatura & 1 & 2,1 \\
\hline Umidade & 1 & 2,1 \\
\hline
\end{tabular}

Tabela 4 Fatores predisponentes individuais associados à alteração vocal

\begin{tabular}{llc}
\hline \multicolumn{1}{c}{ Variável } & $n$ & $\%$ \\
\hline Alergia & 7 & 14,9 \\
Infecção do trato respiratório & 6 & 12,8 \\
Rinite/sinusite & 5 & 10,6 \\
Asma & 3 & 6,4 \\
Estresse & 3 & 6,4 \\
Refluxo & 3 & 6,4 \\
Perda auditiva & 2 & 4,3 \\
Sexo feminino & 2 & 4,3 \\
Ansiedade & 2 & 4,3 \\
Doença autorrelatada & 2 & 4,3 \\
Doenças gastrointestinais & 2 & 4,3 \\
Idade & 2 & 4,3 \\
Poucas horas diárias de sono & 2 & 4,3 \\
Limpeza frequente da garganta & 2 & 4,3 \\
Ausência de check-up anual & 1 & 2,1 \\
Consumo de café & 1 & 2,1 \\
Depressão & 1 & 2,1 \\
Desidratação & 1 & 2,1 \\
Histórico familiar de disfonia & 1 & 2,1 \\
Humor & 1 & 2,1 \\
Problemas hormonais & 1 & 2,1 \\
Transtorno mental & 1 & 2,1 \\
\hline
\end{tabular}




\section{Discussão}

Este estudo buscou identificar, na literatura, evidências da relação entre trabalho e distúrbio de voz. Os resultados demonstraram que profissionais que utilizam a voz no ambiente de trabalho, em sua maioria professores, podem ter alteração vocal em decorrência das condições desfavoráveis às quais estão expostos, sejam relacionadas aos aspectos pessoais (a exemplo das alergias), mas principalmente, relacionadas a fatores do ambiente (como ruído, acústica inapropriada, poeira/pó de giz/fumaça) e aspectos organizacionais do processo de trabalho, como extensa carga horária, número excessivo de alunos por turma e uso intenso da voz.

\section{Desenho de estudo das publicações}

Dados de prevalência e fatores associados elencados nesta pesquisa foram produzidos por estudos observacionais. Neste tipo de estudo não se objetiva intervir sobre o problema em questão, mas apenas observar a distribuição do evento de interesse e de seus fatores condicionantes ${ }^{16}$. Predominaram os estudos transversais (mais de $3 / 4$ da produção identificada), os quais empregaram questionários e/ou protocolos estruturados para avaliar as alterações vocais. Alguns estudos também utilizaram avaliação fonoaudiológica ou otorrinolaringológica para complementar e confirmar os achados. Dois artigos encontrados nesta pesquisa eram do tipo híbrido.

A categoria de desenhos híbridos corresponde àqueles estudos realizados em dois momentos diversos num período de tempo. Um estudo híbrido transversal/ intervenção ${ }^{15}$ comparou, primeiramente, a referência de rouquidão por meio de questionário em um grupo de professores $(79,5 \%)$ e funcionários administrativos de uma empresa $(29,3 \%)^{17}$, e, posteriormente, realizou uma intervenção pós-teste com o mesmo grupo, aplicando novamente o mesmo questionário, permitindo observar melhora significativa no conhecimento sobre higiene vocal ${ }^{17}$. Outro estudo, do tipo híbrido seguimento seccional ${ }^{15}$, avaliou um grupo de professores num período de 12 anos, considerando prevalência inicial (12\%) e final (29\%) de sintomas vocais ${ }^{18}$.

Os estudos observacionais transversais avaliam os eventos de interesse (exposição e desfecho) num determinado momento, ou seja, são pontuais no tempo. Logo, não há garantia de antecedência temporal entre causa e efeito ${ }^{16}$. Como a maioria dos estudos realizados utilizou esse tipo de abordagem, as evidências obtidas deparam-se com essa limitação: não permitem estabelecer, com clareza, a sequência temporal dos eventos analisados.

A produção brasileira teve percentual significativo em periódicos internacionais (quase metade), o que mostra uma boa visibilidade dos estudos conduzidos no Brasil no cenário internacional.

\section{Número de sujeitos}

Registrou-se uma grande variação no tamanho das populações de estudo. Para que o resultado estatístico seja válido, a seleção do tamanho da amostra deve seguir procedimentos específicos. Quando o estudo pretende comparar duas variáveis, um desfecho ou uma exposição em dois grupos, a definição amostral deve considerar esse fator. Em geral, isso implica definir um tamanho amostral maior do que seria necessário para avaliar um desfecho, sem considerar a condição de exposição e não exposição. Amostras pequenas podem dificultar a avaliação de associações, por não apresentarem poder estatístico suficiente. Portanto, muitas vezes, a não observação de associação entre exposição e desfecho pode ser imputada mais à impossibilidade de apreender diferenças reais existentes do que à ausência concreta de tais diferenças. Isto tende a produzir resultados negativos ou falta de associação.

O levantamento realizado mostrou prevalência média de $44,2 \%$ dos profissionais que relataram ter ou já ter tido alteração vocal. Fortes et al. $(2007)^{19}$ apresentam dados similares aos encontrados na sumarização dos trabalhos analisados, evidenciando altas prevalências de distúrbio de voz nas populações investigadas. Nesta revisão, as prevalências mais elevadas foram encontradas em professores, categoria profissional que se destacou em função do contingente elevado de trabalhadores, da grande tradição no uso intenso da voz e das condições de trabalho a qual está exposta.

\section{Dados Sociodemográficos}

Em relação à idade, no call center, grande parte dos teleoperadores apresentou entre 21 e 30 anos $^{20}$. Esta categoria profissional é de jovens trabalhadores, conforme demonstra estudo $(27,2 \text { anos })^{21}$. Professores com e sem problemas de voz autorrelatados apresentam médias maiores de idade, variando de 31,5 a 40,5 $\operatorname{anos}^{22}$. O mesmo ocorreu com instrutores de fitness $(37,2 \text { anos })^{23}$ e cantores $(42,1 \text { anos })^{24}$, valores próximos à média encontrada nesta pesquisa (40,6 anos). Os resultados citados podem decorrer das diferenças das profissões: professores e cantores geralmente optam por uma carreira e ficam nela por mais tempo, enquanto os operadores de telesserviço encontram nessa área uma oportunidade de emprego temporária, de ingresso rápido no mercado de trabalho, que não exige experiência ou qualificação profissional.

Em relação ao sexo, os estudos foram majoritariamente realizados com ambos. No entanto, as mulheres se destacaram, representando a maioria da população 
docente nos estudos analisados, sendo o sexo que mais apresentou alteração vocal. Outros estudos mostraram resultado semelhante. Em São Paulo, em um estudo com 97 professores, 92,3\% eram mulheres ${ }^{25}$. Em 2015, em estudo com 2.044 pastores luteranos, a maioria homens (53\%), a prevalência de diagnóstico de distúrbio de voz foi maior entre as mulheres (31\%), em relação aos homens $(19 \%)^{26}$. Estudo realizado na Nigéria demonstrou que das 275 participantes do sexo feminino, 126 apresentaram disfonia ${ }^{27}$.

Em geral, professoras do sexo feminino apresentaram mais problemas vocais do que professores do sexo masculino, o que pode estar relacionado às diferenças nos postos de trabalho, aos fatores biológicos da laringe ${ }^{28}$, ou ainda, aos fatores culturais.

Geralmente homens começam a perder a eficiência da voz por volta dos 60 anos, enquanto, entre as mulheres, isso ocorre a partir dos 50 anos. A faixa de maior eficiência vocal ocorre entre 25 e 45 anos $^{16}$, de forma que a média de 40,6 anos observada neste estudo se encontra dentro desta faixa e poderia ser um fator protetor predisponente. Contudo, ainda assim, professores do sexo masculino também apresentaram altas prevalências de alteração vocal, evidenciando que os fatores do ambiente e da organização do trabalho são potenciais agentes desencadeadores do distúrbio de voz.

\section{Profissão}

Pesquisas avaliaram, por meio da aplicação de questionários, a autorreferência de alteração vocal em grupos de professores, que variou de $10,4 \%$ a $87,3 \%^{29,30}$. Estudos caso-controle compararam a presença de transtorno da voz entre professores e grupo controle, evidenciando alteração vocal mais prevalente em docentes ${ }^{31,32}$. Monitoramento de voz de 57 professores revelou uma porcentagem média de uso de voz ocupacional $(29,9 \%)$ maior do que do uso não ocupacional $(14,4 \% \text {. })^{33}$. Esses achados confirmam que os profissionais da voz a utilizam por mais tempo e em maior intensidade na rotina de trabalho, colaborando para o surgimento de um DVRT.

Dentre os estudos selecionados, um apresentou desenho longitudinal do tipo coorte, com acompanhamento de 469 professores, avaliados em dois momentos (2011 e 2014), cuja incidência de alteração vocal autorreferida estimada foi de $17,0 \%{ }^{34}$.

Além de docentes, os estudos evidenciaram alterações vocais em outras categorias profissionais, embora com menor foco na literatura. Pesquisas com religiosos confirmaram o autorrelato frequente de alteração vocal. Em 2015 e 2016 dois estudos revelaram que, respectivamente, $21 \%$ e $47,8 \%$ dos padres relataram problemas vocais ${ }^{26,35}$. Outras duas pesquisas demostraram que $85,6 \%$ dos padres ${ }^{36}$ e $47 \%$ das pastoras ${ }^{37}$ relataram desordem vocal. É importante salientar que foram feitos estudos com diferentes religiões (luterana, católica e Mar Thoma) e em todas identificou-se alteração vocal significativa ${ }^{26,35,37}$.

Trabalhar em ambiente estressante, sob pressão para atingir metas é uma realidade na rotina dos operadores de telemarketing ${ }^{38}$. Pesquisa com teleoperadores encontrou queixa de distúrbio de voz em $43,1 \%$ dos profissionais estudados ${ }^{39}$. Quando comparados com trabalhadores administrativos de uma mesma empresa, os teleoperadores autorreferiram sintomas vocais mais frequentemente ${ }^{40}$. O monitoramento do tempo de fonação de 92 operadores de call center durante o trabalho identificou tempo médio de $14,7 \%$, número maior do que a fonação durante horas não trabalhadas $(6,2 \%)^{41}$. Esses achados evidenciam que a atividade de teleatendimento envolve grande risco para o desenvolvimento de um DVRT, devido ao uso intenso da voz no trabalho.

Outros trabalhadores, menos abordados nos artigos levantados, podem apresentar problemas de voz. Cantar sem as devidas orientações pode levar a um "abuso vocal" 42,43 , aumentando o risco de sintomas vocais e, consequentemente, de DVRT. Ressalta-se, contudo, que a maior sobrecarga vocal não deve ser entendida, necessariamente, como abuso pessoal, mas como uma imposição do trabalho sob condições precárias ${ }^{8}$. Instrutores de fitness autorrelataram elevada prevalência de sintomas de alteração de voz crônica $(70,9 \%)^{23}$. Em atores, avaliação otorrinolaringológica revelou que $59 \%$ dos profissionais tinham achados na laringe de hiperfunção vocal ${ }^{44}$. Estudo com vendedores observou que $25 \%$ deles relataram alteração de voz ${ }^{45}$.

\section{Fatores associados à alteração vocal}

Nesta revisão, os resultados mostraram que $63,8 \%$ dos artigos referiram fatores organizacionais e ambientais e $42,6 \%$ fatores pessoais como agravantes de problemas de voz. O DVRT manifesta-se por presença de sinais e sintomas que podem variar de acordo com a gravidade do quadro clínico.

\section{Fatores do ambiente de trabalho}

Foram encontradas alterações vocais em categorias que não utilizavam a voz profissionalmente como principal instrumento de trabalho. Em estudo realizado com trabalhadores metalúrgicos, 9,6\% relataram ter tido problemas de voz no presente ou no passado ${ }^{46}$. Esses achados evidenciam o quanto fatores de exposição relacionados ao ambiente também podem contribuir para o DVRT, independentemente do uso da voz como instrumento de trabalho. 
O ruído foi o fator associado a alterações vocais relatado com maior frequência. Estudo realizado com professores revelou o barulho dentro da sala de aula como fator mais percebido quando comparado ao ruído externo ${ }^{47}$. A queixa foi relatada também por teleoperadores $^{39}$, vendedores ${ }^{45}$, além de metalúrgicos ${ }^{46}$. Essa recorrência de queixas acerca do ruído remete a outro fator de risco que também se mostrou um dos fatores mais frequentes: a acústica insatisfatória.

O ruído é a produção sonora que causa incômodo e se torna indesejável, gerando desconforto ao sistema auditivo. A exposição ao ruído escolar ${ }^{48}$ é, na maioria das vezes, acima da média permitida pela Norma Brasileira NBR $10.152^{49}$, que estabelece valores máximos para o ruído nas salas de aula. Estudos que utilizaram análise objetiva, por meio da mensuração do nível de ruído $(4,3 \%)$, para verificar o impacto na voz de professores, encontraram valores acima do limite ideal de conforto acústico ${ }^{50,51}$. Os resultados mostram que o ruído pode ser prejudicial para a qualidade vocal de trabalhadores que utilizam a voz na atividade laboral, elevando o esforço durante o exercício profissional.

Outro fator bastante citado foi a exposição a giz, poeira e fumaça. Na literatura encontrou-se relação destes fatores com alteração do mecanismo vocal, devido à irritação da região nasal e laríngea ao inalar microrganismos presentes na poeira e também pela irritação/inflamação por ela gerada ${ }^{52}$. Pesquisa realizada com metalúrgicos também referiu produtos químicos e poeira como fatores que podem ter relação com o DVRT $^{46}$ mesmo se tratando de uma profissão que não utiliza a voz como instrumento de trabalho. Esta exposição, quando frequente, pode levar ao desenvolvimento de doenças respiratórias e problemas alérgicos ${ }^{53,54}$.

\section{Fatores organizacionais e do processo de trabalho}

A extensa carga horária foi o fator mais relatado (17\%) nos estudos revisados. Sua relevância é evidente, posto que quanto maior é o tempo utilizando a voz, maior será o desgaste produzido no aparelho fonador, elevando a probabilidade de alteração vocal. O uso intenso da voz foi outro fator relevante. Vários autores referem este como o principal fator para o aparecimento do distúrbio de voz ${ }^{55}$, pois o uso em excesso pode gerar sobrecarga vocal, influenciando o funcionamento das pregas vocais.

No levantamento realizado, identificou-se associação entre tempo de trabalho ${ }^{53}$, número de alunos por turma ${ }^{54}$ e disfonia. Outros estudos, no entanto, não encontraram associação destes fatores com alteração vocal ${ }^{55}$. Isto pode estar relacionado com a forma de avaliação utilizada nos estudos, visto que muitas vezes o profissional não estabelece relação entre o adoecimento e seu trabalho. Além disto, outros fatores associados ao ambiente de trabalho e às condições nas quais a voz é utilizada (adequação da acústica, uso ou não de microfone, dentre outros fatores) podem reduzir ou aumentar o risco relativo ao número de alunos em sala. Portanto, estes são elementos que devem ser considerados quando ao se analisar o número de alunos e sua relação com o DVRT. Independentemente disto, ressalta-se a gravidade do problema, uma vez que a quantidade de professores que autorreferiram transtornos de voz foi significativa.

\section{Fatores predisponentes}

Alergias, infecção do trato respiratório e rinite/ sinusite foram os fatores mais frequentes nesta pesquisa. Estudos descritos na literatura também associaram tais fatores como agravantes de problemas de voz ${ }^{56,57}$. Pesquisas mostram que os professores apresentam maior risco de ter alergias e problemas respiratórios ${ }^{57}$ por estarem expostos, em sua maioria, a produtos químicos, como poeira, pó de giz, produtos de limpeza, ou ainda a tinta do pincel atômico utilizado no quadro branco. No entanto, estudo com padres também relatou alergias e problemas respiratórios como fatores que interferem na qualidade vocal $^{37}$. Isto mostra que, além de associados a características predisponentes, as condições do ambiente também favorecem os agravos respiratórios ${ }^{58}$.

O estresse em trabalhadores que usam a voz como instrumento de trabalho contribui para distúrbios da voz e pode impactar o desempenho profissional, resultando, adicionalmente, em problemas psicológicos. Quando o problema de voz é percebido e comentado de forma negativa por outras pessoas, a consequência pode ser o isolamento social, o desenvolvimento de ansiedade acerca de sua vida laboral e as frustrações pela limitação que a voz alterada pode causar $^{59}$. Estudo realizado no Brasil demonstrou maior incidência de distúrbio de voz em professores que apresentaram transtorno mental ${ }^{36}$. Essa instabilidade emocional prejudica não apenas a qualidade vocal, como também a saúde em geral, podendo levar a episódios de depressão.

Estudo realizado no Egito $^{17}$ revelou que professores com distúrbios de voz apresentaram maior sofrimento psicológico ou ansiedade em comparação a professores sem problemas de voz. Ansiedade e depressão podem ser fatores tanto relacionados à organização e processo de trabalho quanto predisponentes. Contudo, mesmo que o indivíduo tenha um fator predisponente, as condições de trabalho exaustivas, que comprometem seu desempenho vocal, são determinantes do adoecimento. Essa é a ideia da concausalidade, classificada, segundo Schilling, no grupo III, ou seja, o trabalho como agravante de condição pré-existente ${ }^{10}$. 
Da mesma forma, fatores individuais por si só não provocam um DVRT. É necessário o componente trabalho para dispará-lo. Deste modo, mesmo não sendo a causa única, o trabalho pode agravar a doença já existente ou colaborar para o surgimento de um DVRT ${ }^{10}$.

\section{Tipos de avaliação}

A autoavaliação obtida por meio da aplicação de questionários e protocolos foi predominante nos resultados encontrados nesta revisão. Este tipo de avaliação tem se tornado cada vez mais frequente em pesquisas da área.

A avaliação perceptivo-auditiva foi outro método utilizado para avaliar a qualidade vocal e seus desvios, sendo instrumento padrão de referência na clínica fonoaudiológica ${ }^{16}$. Embora de natureza subjetiva, posto que é realizada pelo ouvido do clínico, é considerada importante parâmetro de avaliação da voz, uma vez que identifica padrões vocais por meio de escalas padronizadas, como a japonesa GRBAS (Grade, Roughness, Breathiness, Asthenia, Strain - Grau de Desvio Vocal, Rugosidade, Soprosidade, Astenia e Tensão) e a analógico-visual americana Consensus Auditory -Perceptual Evaluation of Voice (CAPE-V) ${ }^{60}$.

Também realizada pelo fonoaudiólogo, a análise acústica é um procedimento que permite extrair indicadores objetivos das características vocais, captados e analisados por meio de um programa de computador ${ }^{16}$. É uma ferramenta auxiliar à avaliação perceptivo-auditiva e pouco sensível a desvios muito acentuados da voz ${ }^{16}$.

A avaliação otorrinolaringológica, por sua vez, objetiva realizar o diagnóstico médico por meio da "visibilização da região laringofaríngea"4. Por ser considerada um exame invasivo, somente médicos (preferencialmente otorrinolaringologistas) podem realizá-la. Apresenta nível mais sofisticado, em termos de diagnóstico vocal, uma vez que identifica a presença ou ausência de lesões laríngeas, embora tenha certo grau de subjetividade, visto que é realizada por intermédio da visão do médico.

Deve-se registrar, contudo, que submeter uma população sem sintomas ou queixas a diagnósticos médicos ou fonoaudiológicos, além dos elevados custos envolvidos, poderia expor os trabalhadores a procedimentos invasivos desnecessários. Para efeitos epidemiológicos, a autoavaliação pode ser um instrumento de varredura eficaz, de identificação dos casos e, portanto, instrumento utilizado para planejar políticas públicas.

Ausência de consenso na definição do desfecho de interesse e dos métodos para sua investigação

Um aspecto de grande importância a ser destacado nesta revisão é a diversidade de definições e conceitos do desfecho de interesse e dos métodos e instrumentos empregados para mensurá-los. Disto decorrem as grandes dificuldades em comparar resultados obtidos e confirmar ou não a hipótese de sua associação com o trabalho. Os estudos identificados nesta revisão apresentam definições diferentes, além de utilizarem distintos métodos de avaliação do distúrbio de voz, impossibilitando comparar as estimativas de prevalência e incidência e as medidas de associação com fatores ocupacionais. Apesar das referidas limitações, ainda assim é possível verificar a elevada prevalência de distúrbio de voz, associado a fatores relacionados ao ambiente e à organização de trabalho.

\section{Conclusão}

Este artigo reuniu resultados que sustentam a hipótese de relação entre trabalho e distúrbios vocais, uma vez que trabalhadores que usavam a voz profissionalmente apresentaram, com consistência, maior frequência de um DVRT. Os achados provêm de estudos transversais que, apesar de suas fragilidades, podem contribuir para fortalecer o nexo entre distúrbio de voz e trabalho, confirmando as elevadas prevalências desse agravo, especialmente em professores do ensino fundamental, de meia idade e de ambos os sexos. O DVRT associou-se fundamentalmente a fatores do ambiente e da organização do trabalho, especialmente ao ruído e à extensa carga horária, como também a fatores predisponentes concausais, a exemplo de alergias. O instrumento de avaliação para estabelecer o nexo causal mais frequentemente utilizado foi a autorreferência de alteração vocal, geralmente feita por meio de questionários e protocolos. Parte das referências levantadas foi utilizada na atualização e revisão do protocolo DVRT, publicado pelo Ministério da Saúde em julho de 2018. Poucas pesquisas sobre a incidência de DVRT foram encontradas, de forma que é necessário conduzir estudos longitudinais (coorte) para avaliar a ocorrência de novos casos de alteração vocal em determinados grupos profissionais e sua relação com o trabalho, de modo a produzir evidências mais consistentes.

Em tempo: Entre a data de aprovação do artigo e sua efetiva publicação, o Ministério da Saúde, por meio da Coordenação Geral de Saúde do Trabalhador (CGSAT/DSAT/SVS/MS), anunciou a atualização da lista de doenças relacionadas ao trabalho (LDRT), na $5^{\mathrm{a}}$ Reunião Ordinária da Comissão Intergestores Tripartite, realizada em 30 de julho de 2020, com a inclusão do distúrbio de voz. Aguardam-se a publicação do documento e a respectiva portaria. O anúncio pode ser conferido no site: https://www.youtube. $\mathrm{com} /$ watch?v=gPCV_Fk5kKk. Em 01 de setembro 
de 2020, foi publicada no Diário Oficial da União a Portaria GM/MS no 2.309/2020 com a nova LDRT. Um dia após, a Portaria GM/MS n ${ }^{\circ} 2.345 / 20$, revogou a anterior, tornando-a sem efeito. Por meio da subsequente Portaria GM/MS n ${ }^{0}$ 2.384/2020, a LDRT de 21 anos atrás é repristinada (Portaria GM/MS Portaria $\left.n^{\circ} 1.339 / 1999\right)$, voltando a vigorar. Nela, não está incluído o DVRT.

\section{Contribuições de autoria}

Jesus MTA, Guimarães SF, Araujo TM e Vaz MLM contribuíram igualmente nas diversas fases da investigação e da redação do artigo, as quais incluíram coleta de dados, análise, redação, revisão e aprovação do texto para publicação. Todos assumem integral responsabilidade sobre o estudo e o conteúdo aqui publicado.

\section{Referências}

1. Organização Pan-Americana da Saúde. Saúde do Trabalhador [Internet]. [citado em 7 ago 2017]. Disponível em: https://www.paho.org/bra/index. php?option $=$ com_content $\&$ view $=$ article\&id $=378: \mathrm{s}$ aude-do-trabalhador\&Itemid $=685$.

2. Brasil. Ministério da Saúde. Portaria $\mathrm{n}^{0} 1.823$, de 23 de agosto de 2012. Diário Oficial da União [Internet]. 24 ago 2012 [citado em 21 ago 2020]. Disponível em; http://bvsms.saude.gov.br/bvs/ saudelegis/gm/2012/prt1823_23_08_2012.html.

3. Brasil. Lei no 8.080, de 19 de setembro de 1990. Diário Oficial da União [Internet]. 20 set 1990 [citado em 21 ago 2020]; 1:18055. Disponível em: http://www.planalto.gov.br/ccivil_03/leis/l8080.htm.

4. Brasil. Ministério da Saúde. Distúrbio de voz relacionado ao trabalho [Internet]. 2018 [citado em 24 set 2018]. Disponível em: http://bvsms.saude. gov.br/bvs/publicacoes/disturbio_voz_relacionado_ trabalho_dvrt.pdf

5. 5. Ferreira LP, Märtz MLW. Distúrbio de voz relacionado ao trabalho: a experiência dos Cerest. BEPA. 2010;7(76):13-9.

6. Ferreira LP, Bernardi APA. Distúrbio de voz relacionado ao trabalho: resgate histórico. Disturb Comun. 2011;23(2):233-6.

7. Ferracciu CCS, Almeida MS. O distúrbio de voz relacionado ao trabalho do professor e a legislação atual. Rev CEFAC. 2014;16(2):628-33.

8. Masson MLV, Ferrite S, Pereira LMA, Ferreira LP, Araújo TM. Em busca do reconhecimento do distúrbio de voz como doença relacionada ao trabalho: movimento histórico-político. Cienc Saude Coletiva. 2017;24(3):805-16

9. Costa HO. Distúrbios da voz relacionados com o trabalho. In: Mendes R, organizador. Patologia do trabalho. São Paulo: Atheneu; 2003. p. 1283-94.

10. Schilling RSF. More effective prevention in occupational health practice? J Soc Occup Med. 1984;34(3):71-9.

11. Przysiezny PE, Przysiezny LTS. Work-related voice disorder. Braz J Otorhinolaryngol. 2015;81(2):202-11.

12. Brasil. Ministério da Saúde. Doenças relacionadas ao trabalho: manual de procedimentos para os serviços de saúde. Brasília, DF: Ministério da Saúde; 2001.

13. Williams NR. Occupational groups at risk of voice disorders: a review of the literature. Occup Med. 2003;53(7):456-60.

14. Jardim R, Barreto SM, Assunção AA. Voice disorder: case definition and prevalence in teachers. Rev Bras Epidemiol. 2007;10(4):625-36.

15. Klein CH, Bloch KV. Estudos seccionais. In: Medronho RA, editor. Epidemiologia. 2a ed. São Paulo: Atheneu; 2011. p. 193-220.

16. Behlau M. Voz: o livro do especialista: volume 1. Rio de Janeiro: Revinter; 2001.

17. Bolbol SA, Zalat MM, Hammam RAM, Elnakeb NL. Risk factors of voice disorders and impact of vocal hygiene awareness program among teachers in public schools in Egypt. J Voice. 2017;31(2):251.e9-e16.

18. Simberg S, Sala E, Vehmas K, Laine A. Changes in the prevalence of vocal symptoms among teachers during a twelve-year period. J Voice. 2005;19:95-102.

19. Fortes FSG, Imamura R, Tsuji DH, Sennes LU. Perfil dos profissionais da voz com queixas vocais atendidos em um centro terciário de saúde. Rev Bras Otorrinolaringol. 2007;73(1):27-31.

20. Piwowarczyk TC, Oliveira G, Lourenço L, Behlau M. Vocal symptoms, voice activity, and participation profile and professional performance of call center operators. J Voice. 2012;26(2):194-200.

21. Ben-David BM, Michal I. Voice changes in real speaking situations during a day, with and without vocal loading: assessing call center operators. J Voice. 2016;30(2):247.e1-11.

22. Rodrigues G, Zambon F, Mathieson L, Behlau M. Vocal tract discomfort in teachers: its relationship to self-reported voice disorders. J Voice. 2013;27(4):473-80.

23. Rumbach AF. Vocal problems of group fitness instructors: prevalence of self-reported sensory and auditory-perceptual voice symptoms and the need for preventative education and training. J Voice. 2013;27(4):524.e11-21. 
24. Rosa M, Behlau M. Mapping of vocal risk in amateur choir. J Voice. 2017;31(1):118.e1-11.

25. 25. Martinello JG, Lauris JRP, Brasolotto AG. Psychometric assessments of life quality and voice for teachers within the municipal system, in Bauru, SP, Brazil. J Appl Oral Sci. 2011;19(6):573-8.

26. Hagelberg AM, Simberg S. Prevalence of voice problems in priests and some risk factors contributing to them. J Voice. 2015;29(3):389.e11-8.

27. Akinbode R, Lam KBH, Ayres JG, Sadhra S. Voice disorders in Nigerian primary school teachers. J Occup Med. 2014;64(5):382-6.

28. Butler JE, Hammond TH, Gray SD. Genderrelated differences of hialuronic acid distribution in the human vocal fold. Laryngoscope. 2001;111(5):907-11.

29. Moy FM, Hoe VCW, Hairi NN, Chu AHY, Bulgiba A, Koh D. Determinants and effects of voice disorders among secondary school teachers in peninsular Malaysia using a validated Malay version of VHI-10. PLoS One. 2015;10(11):e0141963.

30. Alves LP, Araujo LTR, Xavier Neto JA. Prevalência de queixas vocais e estudo de fatores associados em uma amostra de professores de ensino fundamental em Maceió, Alagoas, Brasil. Rev Bras Saude Ocup. 2010;35(121):168-75.

31. Giannini SPP, Latorre MRDO, Fischer FM, Ghirardi ACAM, Ferreira LP. Teachers' voice disorders and loss of work ability: a case-control study. J Voice. 2015;29(2):209-17.

32. Tavares ELM, Martins RHG. Vocal evaluation in teachers with or without symptoms. J Voice. 2007;21(4):407-14.

33. Hunter EJ, Titze IR. Variations in intensity, fundamental frequency, and voicing for teachers in occupational versus non occupational settings. J Speech Lang Hear Res. 2010;53(4):862-75.

34. Rocha LM, Lima BS, Amaral PL, Behlau M, Souza LDM. Risk factors for the incidence of perceived voice disorders in elementary and middle school teachers. J Voice. 2017;1(2):258.e7-12.

35. Devadas U, Jose N, Gunjawate D. Prevalence and influencing risk factors of voice problems in priests in Kerala. J Voice. 2016;30(6):771.e27-32.

36. Hocevar-Boltezar I. Prevalence and risk factors for voice problems in priests. Wien Klin Wochenschr. 2009;121:276-81.

37. Middleton RL, Hinton VA. A preliminary investigation of the vocal behaviors and characteristics of female pastors. J Voice. 2009;23(5):594-602.

38. Amorim GO, Bommarito S, Kanashiro CA, Chiari BM. The vocal behavior of telemarketing operators before and after a working day. J Soc Bras Fonoaudiol. 2011;23(2):170-6.

39. Santos CT, Santos C, Lopes LW, Silva POC, LimaSilva MFB. Relationship between working and voice conditions self-reported by telemarketers of an emergency call center. CoDAS. 2016;28(5):583-94.

40. Rechenberg L, Goulart BNG, Roithmann R. Impact of call center work in subjective voice symptoms and complaints: an analytic study. J Soc Bras Fonoaudiol. 2011;23(4):301-7.

41. Cantarella G, Iofrida E, Boria P, Giordano S, Binatti O, Pignataro L et al. Ambulatory phonation monitoring in a sample of 92 call center operators. J Voice. 2014;28(3):393.e1-6.

42. Dassie-Leite AP, Duprat AC, Busch R. Comparação de hábitos de bem-estar vocal entre cantores líricos e populares. Rev CEFAC. 2011;13(1):123-31.

43. Van Houtte E, Van Lierde K, D’Haeseleer E, Claeys $\mathrm{S}$. The prevalence of laryngeal pathology in a treatment-seeking population with dysphonia. Laryngoscope. 2010;120(2):30612.

44. Lerner MZ, Paskhover B, Acton L, Young N. Voice disorders in actors. J Voice. 2013;27(6):705-8.

45. Ferreira LP, Luciano P, Akutsu CM. Condições de produção vocal de vendedores de móveis e eletrodomésticos: correlação entre questões de saúde, hábitos e sintomas vocais. Rev CEFAC. 2008;10(4):528-35.

46. Ubrig-Zancanella MT, Behlau M. Relação entre ambiente de trabalho e alteração vocal em trabalhadores metalúrgicos. Rev Soc Bras Fonoaudiol. 2010;15(1):72-9.

47. Mathieson L, Hirani SP, Epstein R, Baken RJ, Wood G, Rubin JS. Laryngeal Manual therapy: a preliminary study to examine its treatment effects in the management of muscle tension dysphonia. J Voice. 2009;23(3):353-66.

48. Cutiva LCC, Burdorf A. Effects of noise and acoustics in schools on vocal health in teachers. Noise Health. 2015;17(74):17-22.

49. Associação Brasileira de Normas Técnicas. NBR 10.152. Acústica: níveis de pressão sonora em ambientes internos a edificações. 2a ed. Rio de Janeiro: ABNT; 2017.

50. Mendes ALF, Lucena BTL, Araújo AMG, Melo LPF Lopes LW, Silva MFBL. Voz do professor: sintomas de desconforto do trato vocal, intensidade vocal e ruído em sala de aula. CoDAS. 2016;28(2):168-75.

51. Simões-Zenari M, Bitar ML, Nemr NK. The effect of noise on the voice of preschool institution educators. Rev Saude Publica. 2012;46(4):657-64.

52. Sataloff RT, Spiegel JR. Care of the professional voice. Otolaryngol Clin North Am. 1991;24:1093-124.

53. Silvany Neto AM, Araújo TM, Dutra F, Azi G, Alves R, Kavalkievcz C et al. Condições de trabalho e saúde de professores da rede particular de ensino de Salvador, Bahia. Rev Saude Publica. 2000;24:42-56.

54. Ferreira LP, Giannini SPP, Figueira S, Silva EE, Karmann DF, Souza TMT. Condições de produção vocal de professores da prefeitura do município de São Paulo. Dist Comun. 2003;14(2):275-307. 
55. Leite APD, Carnevale LB, Rocha HL, Pereira CA, Lacerda Filho L. Relação entre autoavaliação vocal e dados da avaliação clínica em indivíduos disfônicos. Rev CEFAC. 2015;17(1)44-51.

56. Smith E, Gray SD, Dove H, Kirchner L, Heras H. Frequency and effects of teachers voice problems. J Voice. 1997;11(1):81-7.

57. Roy N, Merrill RM, Gray SD, Smith EM. Voice disorders in the general population: prevalence, risk factors, and occupational impact. Laryngoscope. 2005;115(11):1988-95.
58. Fuess VLR, Lorenz MC. Disfonia em professores do ensino municipal: prevalência e fatores de risco. Rev Bras Otorrinolaringol. 2003;69(6):807-12.

59. Lemos S, Rumel D. Ocorrência de disfonia em professores de escolas públicas da rede municipal de ensino de Criciúma-SC. Rev Bras Saude Ocup. 2005;30(112):7-13.

60. Kempster GB, Gerratt BR, Verdolini Abbott K, Barkmeier-Kraemer J, Hillman RE. Consensus auditory-perceptual evaluation of voice: development of a standardized clinical protocol. Am J Speech Lang Pathol. 2009;18(2):124-32. 